\title{
SABERES DOCENTES PARA A CONJUNÇÃO ENTRE HISTÓRIA DA MATEMÁTICA E TECNOLOGIAS DIGITAIS DA INFORMAÇÃO E COMUNICAÇÃO NAS AULAS DE MATEMÁTICA: ANÁLISE DO POTENCIAL DE UM CURSO DE EXTENSÃO
}

\section{TEACHING KNOWLEDGE FOR THE CONJUNCTION BETWEEN HISTORY OF MATHEMATICS AND INFORMATION AND COMMUNICATION DIGITAL TECHNOLOGIES IN MATHEMATICS CLASSES: ANALYSIS OF THE POTENTIAL OF AN EXTENSION COURSE}

\author{
Adriana de Bortoli $1^{1}$
}

\begin{abstract}
RESUMO
Desde a década de 1990 tanto a História da Matemática (HM) como as Tecnologias Digitais da Informação e Comunicação (TDIC) foram incorporadas como possibilidades metodológicas dentro do movimento da Educação Matemática. Adicionalmente, foram desenvolvidas pesquisas direcionadas à formação de professores que ensinam Matemática numa perspectiva a utilizar tais tendências. Contudo, no que se refere a possibilidades de articulações entre essas duas tendências, as pesquisas são recentes e diminutas. Com o objetivo de promover a aproximação entre essas tendências construímos e viabilizamos um curso de extensão para professores que ensinam Matemática na rede pública estadual de ensino da região de Lins (SP), o qual foi encaminhado pelo conceito função. Dessa forma, a partir desse curso, nos propomos neste artigo, apresentar as possíveis manifestações de mobilização de saberes docentes emergentes ao longo do referido curso alicerçadas nas tarefas propostas no decorrer do mesmo. Os dados foram produzidos por meio de observação dos encontros (vídeo gravações e diário de campo), bem como questionários que foram aplicados antes e durante o curso. Ademais foram utilizados como fonte, os registros produzidos pelas participantes ao longo do curso. A análise dos dados ocorreu à luz do referencial adotado: História da Matemática no ensino de Matemática, Tecnologias Digitais da Informação e Comunicação e Formação de Professores. Abaliza-se indícios de mobilização de saberes pedagógicos voltados para uma prática pedagógica que demostra ensejo em empregar a conjunção entre História da Matemática e Tecnologias Digitais da Informação e Comunicação. Adicionalmente, nessa nossa primeira tentativa de articulação entre as duas tendências, pudemos verificar o potencial das ações extensionistas para a formação de professores.
\end{abstract}

Palavras-chave: História da Matemática; Tecnologias Digitais Informação e Comunicação; Formação de Professores; Ensino de Matemática; Funções.

\footnotetext{
${ }^{1}$ Doutora em Educação Matemática pela UNESP de Rio Claro. Professora de Ensino Superior da Faculdade de Tecnologia Professor Antonio Seabra-FATEC de Lins (SP), Brasil. Endereço para correspondência: Estrada Mário Covas Jr, s/n, Vila Guararapes, Lins (SP), Brasil, CEP: 16403-025. E-mail: adrianadebortoli1@hotmail.com
}

(iD) ORCID iD: https://orcid.org/0000-0003-2524-4987. 
Saberes docentes para a conjunção entre história da matemática e tecnologias digitais da informação e comunicação nas aulas de matemática: análise do potencial de um curso de extensão

\begin{abstract}
Since the 1990s, both the History of Mathematics (HM) and Information and Comunication Digital Tecnologies (DICT) have been incorporated as methodological possibilities within the Mathematics Education movement. Additionally, research was developed aimed at training teachers who teach mathematics in a perspective to use such trends. However, with regard to the possibilities of articulation between these two trends, research is recent and small. In order to promote the approximation between these trends, we built and made possible an extension course for teachers who teach mathematics in the state public school system in the region of Lins (SP), which was guided by the function concept. Thus, from this course, we propose in this article, to present the possible manifestations of the mobilization of emerging teaching knowledge throughout the course, based on the tasks proposed during the course. The data were produced by observing the meetings (video recordings and field diaries), as well as questionnaires that were applied before and during the course. Furthermore, the records produced by the participants throughout the course were used as a source. Data analysis took place in the light of the adopted framework: History of Mathematics in the teaching of Mathematics, Information and Comunication Digital Tecnologies and, Teacher Education. Evidence of mobilization of pedagogical knowledge is highlighted, focusing on a pedagogical practice that demonstrates the opportunity to employ the conjunction between History of Mathematics and Information and Communication Technologies. Additionally, in our first attempt at articulation between the two trends, we were able to verify the potential of extension actions for teacher education.
\end{abstract}

Keywords: History of Mathematics; Information and Comunication Digital Tecnologies; Teacher Education; Mathematics teaching; Functions. 


\section{Introdução}

Dentre as pesquisas realizadas em Educação Matemática, muitas são centradas na temática da formação de professores, incluído a essa temática muitas possuem como cerne da investigação o Conhecimento do Professor que Ensina Matemática. Nessa esfera, buscam-se pelos conhecimentos necessários a prática docente referente à Matemática, ou seja, o seu conhecimento especializado.

Segundo Caldatto e Ribeiro (2020), diante desses interesses foram propostos diversos modelos analíticos procedentes dos trabalhos de Shulman (2004), e, de acordo com Charalambous e Like (2018) apud Caldatto e Ribeiro (2020), como um dos principais resultados dessas investigações houve a identificação da estreita relação entre o ensino de alta qualidade e um conhecimento profissional sofisticado.

Assim, pensando em agregar subsídios as investigações sobre o processo de ensino de Matemática propomos a inserção da HM e TDIC, em particular, a articulação entre essas tendências, diante da premissa que HM assim como TDIC podem contribuir no processo de ensino de Matemática, conforme constatamos em diversas pesquisas que passaremos a ponderar a seguir.

Considerando que a História da Matemática (HM) tem sido preconizada para o ensino de Matemática por copiosos autores. Trabalhos nacionais escritos por (MIGUEL; BRITO; MENDES, LUCCHESI 2009; MIGUEL; MIORIN, 2008 e MIGUEL, 1993) e internacionais, publicados por (TZANAKIS; THOMAIDIS, 2000; FURINGHETTI, 2012; MOSVOLD; JAKOBSEN; JANKVIST, 2013; DEJIĆ; MIHAJLOVIĆ, 2014), discutem a importância da HM em diversos níveis de ensino.

Além disso, pesquisas centradas na temática das Tecnologias Digitais Informação e Comunicação (ROMANELLO; MALTEMPI, 2016; SANTOS, 2006), bem como das relações destas na formação de professores são frequentes no campo da Educação Matemática (BORBA; PENTEADO, 2003), de modo que diferentes perspectivas de incorporação dos recursos das tecnologias têm sido evidenciadas.

Quanto as discussões sobre as relações entre a HM e TDIC no ensino da Matemática, no Brasil, podemos considerar que os trabalhos ainda são diminutos². 
Pelo exposto anteriormente, elaboramos um curso de extensão, proposto na formação continuada, como forma de promover uma reflexão acerca das possibilidades de aproximação entre essas tendências. O referido curso foi oferecido a docentes da rede pública estadual de ensino da região de Lins (SP), com a seguinte temática "Relações entre História da Matemática e Tecnologias Digitais da Informação e Comunicação para o ensino de função".

Nas próximas seções apresentaremos considerações mais específicas sobre a metodologia da investigação no qual caracterizamos o referido curso e a coleta de dados para depois discorrer sobre o processo de análise dos dados. Ao final deste artigo é exposto os resultados e, as conclusões da pesquisa.

\section{Procedimentos metodológicos}

O trabalho que temos desenvolvido em alguns projetos, envolve contexto diversificado de pesquisa e extensão. No presente texto apresentamos um estudo exploratório de natureza qualitativa, cujo intento foi verificar possíveis mobilizações de saberes docentes ao longo de um curso de extensão alicerçado na vivência de tarefas matemáticas, encaminhadas pela perspectiva de uso de HM e TDIC de maneira concomitante.

Os dados foram empreendidos por meio de registros produzidos pelas cursistas ao longo do curso, de vídeo gravações das atividades presenciais e também das anotações da pesquisadora registradas em um diário de campo.

O curso aconteceu nas segundas-feiras, em seis encontros de duas horas de duração, e foi realizado em parceria com a Diretoria Regional de Ensino (DE) de LinsSP e a Faculdade de Tecnologia de Lins via proposta de um curso de extensão oferecido pela docente da faculdade, autora deste texto. Dessa forma, reuniu-se além da professora pesquisadora, um estudante do Curso Superior de Tecnologia Análise e Desenvolvimento de Sistemas.

Tivemos dez docentes inscritas, sendo duas funcionárias da Diretoria de Ensino de Lins cuja função era de coordenadoras de núcleo pedagógico (PCNP's), sendo uma PCNP de Tecnologia e outra, PCNP de Matemática e ainda, oito professoras de Educação Básica. Desse total, uma não iniciou o curso, pelo fato de ter assumido um cargo na Diretoria Regional de Ensino e outra não esteve presente até o final do curso, por motivos 
particulares. Todos os nomes das colaboradoras foram substituídos a fim de preservar o anonimato das mesmas.

No primeiro encontro, foi esclarecido as participantes que além do curso, também seria conduzida uma investigação e perguntamos quais delas se disporiam a participar da pesquisa. Todas aceitaram participar e assinaram o termo de consentimento livre e esclarecido.

As atividades foram pensadas a partir de estudos que apontam o uso da História da Matemática na formação do professor, Tzanakis e Thomaidis (2000), diante de questionamentos planteados em Fauvel e van Maanen (2002), que foram apresentados em um texto no qual esclarecem aspectos do papel que a história pode desempenhar na apresentação tanto da Matemática como da Física, e ainda em outras referências.

Outra autora tomada como referência foi Furinghetti (2012), que discute o papel da História da Matemática na Educação Matemática por meio de considerações teóricas evidenciando alguns exemplos de prática em sala de aula e na formação de professores e também, Jankvist (2009), que propõe uma maneira de organizar e estruturar a discussão sobre por que e como usar a História da Matemática no ensino e aprendizagem da matemática, bem como as inter-relações entre os argumentos por usar a história e as abordagens para fazê-lo.

Ancoradas em pesquisas como de Dejić e Mihajlović (2014), que em estudos na Sérvia, investigaram as crenças e atitudes dos professores sobre as possibilidades de usar a História da Matemática em sua prática, elaboramos um aparato empírico caracterizado por questionários fechados que foram aplicados as cursistas antes e durante $\mathrm{o}$ desenvolvimento do curso. Tais questionários buscavam pelo tipo de formação inicial, pelo conhecimento ou não das cursistas sobre HM e TDIC.

Os elementos teóricos em que apoiamo-nos sobre o uso de tecnologias constam de textos como de Richit et al. (2012), que afirmam que vários especialistas buscaram perseverantemente novos horizontes a fim de compreender as interfaces do processo de ensino e aprendizagem com o propósito de instigar os estudantes a aprender, aproximando os conceitos às situações vivenciadas no cotidiano, tornando a aprendizagem mais relevante e significativa ao aluno. Ainda atestam que as tecnologias digitais da informação e da comunicação (TDIC) ganham uma nova relevância e necessidade dentro do ensino. 
Entram nesse contexto os softwares educacionais que, segundo Borba (2010), têm a capacidade de realçar o componente visual da matemática, atribuindo um papel importante à visualização na Educação Matemática, além de proporcionar, principalmente, um feedback instantâneo da ação do aluno sobre o problema.

Nessa mesma perspectiva, Santos (2006) discute que o uso desses recursos tem transformado a maneira de produzir o conhecimento, sendo possível notar uma maior difusão de softwares em sala de aula nos últimos anos.

No que se refere a questões epistemológicas, é possível observar que, com o avanço da internet, novas possibilidades de comunicação surgem para a Educação, em especial à Educação Matemática, por modificar as noções de espaço e de tempo e, principalmente, pelas sutilezas que a linguagem matemática apresenta (SANTOS, 2006).

Diante desses referenciais teóricos conduzimos tais atividades e ao final de cada encontro as cursistas eram convidadas a registrar, em seus cadernos, algumas reflexões acerca das discussões e das temáticas abordadas, bem como levavam alguns questionamentos para serem pensados nas horas de atividades não presenciais.

Como forma de aproximação entre as tendências partimos de trabalhos já realizados. Sousa e Gomes (2020), propõem o apoio a promoção de atividades históricascom-tecnologia como recurso para o ensino de matemática. Nesse texto além de apontar para a conexões entre HM e TDIC também propõem o desenvolvimento de atividades pelo processo de Investigação Matemática (IM). Assim, as autoras realizaram um levantamento pautado em trabalhos acadêmicos como dissertações e artigos científicos para estabelecerem quais elementos seriam importantes na definição de tais atividades na direção de interlocução entre as duas tendências via IM.

Um dos trabalhos orientados por Sousa e apontado no artigo supracitado, consta de uma dissertação de mestrado da autora Vieira (2017) que fornece um produto educacional acerca do assunto funções. Esse material foi um grande referencial para a elaboração desse curso de extensão que abordou a história do conceito de funções na perspectiva de Youschkevith (1976), que recomenda a busca nos períodos: antiguidade, idade média e idade moderna. Desse modo, as atividades propostas no produto educacional da referida dissertação foram usadas no curso proposto 
Logo, o primeiro problema apresentado as cursistas constou do problema da antiguidade, chamado de Plimpton $322^{3}$. Tivemos o cuidado para não assumir os conceitos com a ideia de funcionalidade, sem ter sido mencionado nos problemas trabalhados, como exemplo, ao encaminhar uma busca pelo terceiro lado de um triângulo retângulo (problema de Plimpton 322), apresentamos a ideia de reqlação, a busca pelo terceiro lado sem afirmar que os egípcios conheciam a ideia de funcionalidade. $\mathrm{O}$ desenvolvimento dessa atividade foi conduzido com o uso do aplicativo desmos ${ }^{4}$ como possibilidade de resolução de problemas históricos com o uso de tecnologias. Tal aplicativo foi utilizado como uma calculadora gráfica em que as cursistas puderam usar as tabelas e efetivarem cálculos em buscas dos terceiros lados dos triângulos.

Como exemplos de atividades da idade média, foram apresentados o problema latitude das formas de Nicole Oresme (1323-1386) que adicionalmente foi resolvido pelo app desmos. Esse problema constava de estudos do movimento e representação gráfica desses fenômenos, que inclusive era uma característica de alguns problemas da Idade Média. Ao final encaminhamos o problema da corda vibrante, que foi um dos problemas que apareceu como elemento que exerceu influência no desenvolvimento do conceito de função pela sua representação por meio de uma expressão analítica (VIEIRA, 2017). Tal problema é característico do século XVII, ou seja, Idade Moderna.

Assim, o conceito de função foi trabalhado no sentido de apresentar algumas possibilidades de representação de função: aritmética, geométrica e analítica e de suas possíveis identificações com problemas históricos dos três períodos propostos por Youschkevith (1976), conforme recomenda Vieira (2017).

Referente a análise dos dados, foram categorizados elementos advindos de manifestações docentes no decorrer do desenvolvimento das atividades propostas no curso de extensão a partir de registros ou falas produzidas no decorrer do mesmo.

\section{Análise dos dados e alguns resultados}

O objetivo dessa pesquisa foi caracterizar e analisar os conhecimentos sobre a conjunção entre HM e TDIC, mobilizados num processo de formação continuada

\footnotetext{
${ }^{3}$ O problema de Plimpton 322 consta de uma tábua e nesta existem linhas que segundo Eves 2004 referemse a medidas de lados de triângulos retângulos.

${ }^{4}$ Desmos é um software de calculador gráfico implementado como aplicativo web e para celulares.
} 
desenvolvido a partir de um curso de extensão. Tal objetivo dessa investigação se justifica pela escassez de pesquisas na Educação Matemática que abordam a conjunção entre HM e TDIC, bem como, pelas possibilidades apresentadas nos poucos trabalhos nessa direção, a exemplo de Sousa (2020a).

A dinâmica do curso nos deu a oportunidade de identificar contribuições proporcionadas pela participação no curso de extensão em que ocorreu a mobilização de saberes nos quais destacamos três eixos: a leitura e escrita para o ensino de Matemática, História da Matemática na formação das cursistas e conjunção entre a HM e TDIC.

Nesse texto nos propomos a apresentar os dados referentes ao terceiro eixo que foram selecionados, organizados e analisados a partir do referencial empregado (História da Matemática no ensino, Tecnologia Digitais da Informação e Comunicação, conjunção entre HM e TDIC e Formação de Professores de Matemática), conforme já descrito neste texto.

Dessa maneira, trataremos de trechos extraídos dos questionários ou falas das cursistas nos encontros, que consideramos demonstrar indícios de articulação entre as duas tendências por parte das cursistas. Os questionários contemplaram questões de diversas naturezas, a saber: sobre a formação das cursistas, sobre o contato delas com História da Matemática e Tecnologia da Informação e Comunicação, sobre o conhecimento do assunto funções e sobre articulação/aproximação entre HM e TDIC.

Assim, mencionamos uma pergunta que constava no questionário 5: Que elementos facilitadores para o ensino de Função, no nível da Educação Básica, podemos encontrar, a partir da interação História da Matemática e Tecnologia Digitais da Informação e Comunicação (Desmos)?

A resposta escrita pela cursista Ana foi: utiliza-se a história da matemática como problematizador do conceito novo para que o aluno possa construí-lo de forma mais concreta. Utiliza-se das Tic's no aprendizado das funções para que de forma palpável o aluno possa visualizar os conceitos.

Já a cursista Bia respondeu: Utiliza-se tecnologia para demonstrar as funções de maneira que dá para demonstrar vários gráficos rapidamente, com isso fazer comparações.

Obtivemos da cursista Carla a seguinte consideração: primeiro elemento é a motivação espontânea dos alunos em relação ao uso da tecnologia, seguido da 
Saberes docentes para a conjunção entre história da matemática e tecnologias digitais da informação e comunicação nas aulas de matemática: análise do potencial de um curso de extensão

diversidade de ações que envolve o aplicativo, levando-os a refletir sobre o que utilizar e como fazê-lo para chegar ao produto desejado.

Outra resposta foi advinda da cursista Diane: com a interação há a possibilidade de os alunos participarem ativamente na construção e disseminação do seu conhecimento.

Elaine assim se expressa: usamos a HM como possibilidade de iniciar o conceito com um problema novo para que o aluno possa construí-lo de forma mais concreta. Utiliza-se das Tics no aprendizado das funções para que o aluno possa visualizar os conceitos.

Por essas falas, identificamos indícios de uma aproximação entre as duas tendências e, algumas delas, ainda mencionam o papel de cada tendência. Nota-se que para elas a ideia do uso de tecnologia está ancorada na possibilidade de visualização do objeto matemático.

Observamos pelas respostas que mobilizaram um saber didático do conteúdo. $\mathrm{Na}$ perspectiva de Shulman (2014), e, segundo nossa interpretação, conhecimento de recursos virtuais ligados a estratégias de seus usos. Ainda de acordo com Shulman (2014), o conhecimento pedagógico do conteúdo é de especial interesse, porque identifica os distintos corpos de conhecimento necessários para ensinar.

Outra indagação referente a percepção das cursistas quanto a articulação foi proposta no questionário 6, questão 2 que constou de: $\mathbf{O}$ que o aplicativo de celular acrescentou às atividades que foram apresentadas inicialmente pela História da Matemática?

As respostas que expressam o que a questão buscava são advindas de apenas duas cursistas:

A primeira delas, a cursista Ana respondeu: A utilização de aplicativos facilita em termos de tempo para a resolução, bem como propicia a veracidade da resolução de forma clara.

A segunda, cursista Fátima: foi possível identificar uma ideia mais clara de função presente em problemas apresentados na história da matemática.

Por essas respostas, entendemos que o valor dado as TDIC está principalmente na relação com a maximização de tempo da realização da atividade matemática e como uma maneira de confirmar a resolução. Isso nos mostra uma ampliação do olhar acerca das 
Saberes docentes para a conjunção entre história da matemática e tecnologias digitais da informação e comunicação nas aulas de matemática: análise do potencial de um curso de extensão

tecnologias, agora elas também conseguem perceber a chance de esclarecer o conceito com o auxílio de tecnologias.

Notamos uma mobilização de saber pedagógico de conteúdo no depoimento de Carla, pois demonstra uma preocupação em aclarar o conceito que outrora (pela HM) foi iniciado. De acordo com Shulman:

[...] a chave para distinguir a base de conhecimento para o ensino está na interseção entre conteúdo e pedagogia, na capacidade do professor para transformar o conhecimento de conteúdo que possui em formas que são pedagogicamente poderosas e, mesmo assim, adaptáveis às variações em habilidade e histórico apresentadas pelos alunos. (SHULMAN, 2014, p. 217)

Como considerado pelo autor, é relevante a intersecção entre conteúdo e pedagogia. Assim, consideramos que a cursista diante de tal mobilização também desenvolve conhecimento para o ensino de tal conteúdo.

Outro exemplo interessante deu-se no encontro 6, quando levantamos a seguinte indagação: Que relações podemos estabelecer entre a História da Matemática e TDIC para o ensino de função?

Diane respondeu: os dois são usados para facilitar a visão do aluno. Com a união dos dois eu mostro como foi, eu posso mostrar para ele o uso do aplicativo...

De Bia ouvimos que: É muito melhor utilizar a tecnologia com o aluno porque é algo que ele gosta e chama muito a atenção dele porque é muito difícil chamar a atenção do aluno na sala de aula. Então se você trabalha com algo que é interessante para eles então eles vão prestar mais atenção (...) se o aluno não tem o interesse e a atenção então eu vou ficar a aula inteira explicando um exercício, ai o que eufiz? Peguei uns exercícios enviei no whatsApp e na próxima aula iríamos corrigir aqueles exercícios e fiz isso com o terceiro, deu muito certo.

E ainda, Ana respondeu: Tanto a história quanto a tecnologia elas contribuem para a construção do conhecimento. A história tá posta, então o aluno pode ter acesso pelo conhecimento ali em suas mãos só a leitura. Agora, por exemplo, quando lançou a calculadora a criança digitava a conta lá e está pronto na sua mão. Claro que o certo seria que a criança utilizasse a calculadora quando ela soubesse realizar as operações porque ela deve ter o domínio daquilo para depois procurar os métodos mais fáceis.

A última cursista a se manifestar foi Carla: Vimos professores de geografia utilizando o celular para fazer uma pesquisa de determinado assunto e os alunos podiam 
Saberes docentes para a conjunção entre história da matemática e tecnologias digitais da informação e comunicação nas aulas de matemática: análise do potencial de um curso de extensão

utilizar o celular também para fazer tal pesquisa, tinham aplicativos voltados ao assunto. Mas agora, voltados para a matemática ainda não tinham.

Assim, nota-se a importância de cursos de formação dessa natureza uma vez que ela se apropriou das possibilidades de uso de aplicativos para o ensino de matemática que antes ela não tinha conhecimento. Inclusive vale considerar que essa cursista é PCNP de Matemática, e que pela Diretoria de Ensino replica esses cursos em horas de trabalho pedagógico coletivo com os professores (HTPC) em diversas escolas estaduais da região de Lins.

A partir de tais respostas entendemos que ocorreram manifestações docentes em prol das possibilidades de articulação das duas tendências de ensino, ainda que de uma maneira introdutória, elas foram percebidas.

\section{Considerações finais}

As atividades acerca do conceito de funções foram inspiradas no produto educacional elaborado por Vieira (2017). Tal perspectiva histórica adotada pela autora foi pelos estudos históricos de Youschkevith (1976), pelo viés histórico da antiguidade, idade média e idade moderna. Vale considerar que consta de uma historiografia tradicional.

Como temos por premissa que a escolha historiográfica determina uma série de ações na articulação entre história e ensino (SAITO, 2018), apresentamos as cursistas modos de uso de HM no ensino segundo as concepções de Miguel (1993), e atualizados por Brito; Santos e Teixeira (2009), a saber: 1) De fonte de métodos adequados de ensino de matemática, 2) De instrumento de conscientização epistemológica, 3) Unificador e ético-axiológico, 4) De fonte de motivação, 5) De guia para a discussão filosófica sobre o conhecimento matemático, 6) De instrumento de explicação dos porquês e como fonte de objetivos de ensino e 7) De formalização de conceitos.

Além disso, apresentamos as curistas o referencial teórico metodológico acerca do uso de tecnologias com enfoque nas Tecnologias Digitais (TD). Foi indicado e mostrado as funcionalidades de um aplicativo de celular, desmos, com o intuito de encaminhar e resolver os problemas históricos. 
As análises das gravações e dos questionários, evidenciaram elementos para além de uma historiografia tradicional. Emergiram elementos de aspectos políticos, sociais, ou seja, elementos da história cultural.

Como resultados podemos constatar o que a literatura já nos indica sobre HM na formação docente. Foram poucas (duas) as cursistas que afirmaram ${ }^{5}$ terem frequentado uma disciplina de História da Matemática na graduação. De outra parte, três cursistas tiveram conceitos históricos na formação continuada, porém não um curso de História da Matemática, propriamente dito.

Quanto ao uso de tecnologias, elas relataram terem realizado cursos na formação continuada, inclusive, um desses cursos havia sido ministrado pela autora desse artigo. Contudo, como chances da junção ou articulação das duas temáticas, nunca haviam pensado em tal possibilidade, conforme relatos orais durante o curso.

Logo, pelas manifestações docentes, foi observado indícios de mobilização de saberes pedagógicos voltados para uma prática pedagógica que demostra ensejo em empregar a conjunção entre História da Matemática e Tecnologias Digitais da Informação e Comunicação que foi oportunizado pelo curso de extensão.

Assim, entendemos a importância em promover tais ações, uma vez que Shulman (2014) afirma que dentre os aspectos do raciocínio pedagógico uma das etapas do processo é chamada de nova compreensão e consiste em chegar ao novo começo, à expectativa de que, por meio de atos de ensino "pensados" e "lógicos", o professor atinja uma nova compreensão, tanto dos propósitos e dos conteúdos a serem ensinados como dos alunos e dos próprios processos didáticos.

Adicionalmente, os autores Moita e Andrade (2009) mencionam que as discussões acerca do tripé ensino, pesquisa e extensão baseiam-se no princípio de indissociabilidade dos mesmos, garantindo a pretendida integração desses saberes com a ciência, as características particulares de cada uma das três atividades acadêmicas e a permanente articulação entre elas. Dessa maneira, conforme já mencionado na seção de procedimentos metodológicos deste texto, nosso trabalho que tem envolvido contexto diversificado de pesquisa e extensão apresenta alguns resultados nessa esfera de pensamento.

${ }^{1}$ Esses dados foram obtidos em questionários fechados com questões referentes a formação das cursistas e aplicados antes do início do curso de extensão. 
Saberes docentes para a conjunção entre história da matemática e tecnologias digitais da informação e comunicação nas aulas de matemática: análise do potencial de um curso de extensão

\section{Referências}

BORBA, M.C. Softwares e internet na sala de aula de Matemática. Anais do X Encontro Nacional de Educação Matemática, 2010.

BORBA, M.C.; PENTEADO, M. G. Informática e Educação Matemática. 3.ed. Belo Horizonte: Autêntica, 2003.

BRITO, A. J.; SANTOS, K. E. S.; TEIXEIRA, M. R. G. A história nos planos de ensino de futuros professores de matemática. Horizontes. v.27, n.1, p.115-120, 2009.

CALDATTO, M. E; RIBEIRO, C. M. Especificidades do conhecimento do professor de matemática na e para a formação: uma discussão em torno do programa de complementação pedagógica. Revista Brasileira de Educação. v.5, e250031, p.1-26, 2020.

DEJIĆ, M. MIHAJLOVIĆ, A. M. History of Mathematics and Teaching Mathematics. Teaching Innovations, v.27, n.3, p.15-30, 2014.

FAUVEL, J.; MAANEN, J. van. History in Mathematics Education: The ICMI

Study. Dordrecht/Boston/London: Kluwer Academic Publishers, v. 6, p.201-240, 2002.

FURINGHETTI. F. History and Epistemology in mathematics education. In Vagn Lundsgaard Hansen \& Jeremy Gray (Eds.), History of Mathematics, in Encyclopedia of Life Support Systems (EOLSS), Developed under the Auspices of the UNESCO. Oxford, UK: Eolss Publishers. 2012. Disponível em: https://www.researchgate.net/publication/315665208_2012__History_and_epistemology_in_mathematics_education. Acesso em 30 de out. 2020.

JANKVIST, U.T. A categorization of the "whys" and "hows" of using history in mathematics education. Educ Stud Math, v. 71, p.235-261, 2009. DOI 10.1007/s10649-008-9174-9.

MIGUEL, A. Três Estudos Sobre história. Tese (Doutorado em Educação) Universidade de Campinas. Campinas. 1993. Disponível em: http://repositorio.unicamp.br/handle/REPOSIP/253114. Acesso em 15 nov 2018.

MIGUEL, A. MIORIM, M. A. História na Educação Matemática: propostas e desafios. Belo Horizonte: Autêntica, 2008.

MIGUEL, A.; BRITO, A. J.: LUCCHESI, D.C; MENDES, I. A. História da Matemática em atividades didáticas. 2.ed. São Paulo: Livraria da Física. 2009.

MOITA, F.M. G.S.C; ANDRADE, F.C. B. Ensino-pesquisa-extensão: um exercício de indissociabilidade na pós-graduação. Revista Brasileira de Educação v. 14 n. 41 maio/ago. p. 269- 280, 2009. 
Saberes docentes para a conjunção entre história da matemática e tecnologias digitais da informação e comunicação nas aulas de matemática: análise do potencial de um curso de extensão

MOSVOLD, R. JAKOBSEN. A; JANKVIST, U. T.How Mathematical Knowledge for Teaching May Profitfrom the Study of History of Mathematics. Sci \& Educ. v.23, p.47-60, 2013. https://doi.org/10.1007/s11191-013-9612-7.

POWELL, B. A. (org.). Métodos de pesquisa em educação matemática usando escrita, vídeo e internet. Campinas: Mercado de Letras, 2015.

RICHIT, A., BENITES, V. C., ESCHER, M. A., MISKULIN, R. G. S. Contribuições do software geogebra no estudo de cálculo diferencial e integral: uma experiência com alunos do curso de geologia. 2012. Disponível:

https://revistas.pucsp.br/index.php/IGISP/article/view/8385/6941. Acesso: 03/05/2015.

ROMANELLO, L. A.; MALTEMPI M. V. A utilização do smartphone no ensino de função: a visão dos alunos. In: Encontro Nacional de Educação Matemática, 2016, São Paulo. Anais XII Encontro Nacional de Educação Matemática, p.1-12, 2016.

SAITO, F. A Pesquisa Histórica e Filosófica na Educação Matemática. Revista Eventos Pedagógicos. Edição Especial Temática: História, Filosofia e Educação Matemática Sinop, v. 9, n. 2 (24. ed.), p. 604-618, ago./out. 2018.

SANTOS, S. C. A produção matemática em um ambiente virtual de aprendizagem: O caso da geometria euclidiana espacial. Dissertação (Mestrado em Educação Matemática) - Universidade Estadual Júlio de Mesquita Filho. Rio Claro, 2006. 144 f. Disponível em: https://repositorio.unesp.br/handle/11449/91097. Acesso em: 01 nov. 2020.

SHULMAN, L. S. Conhecimento e ensino: fundamentos para a nova reforma. Cadernos Cenpec| Nova série, [S.1.], v. 4, n. 2, jun. 2014. ISSN 2237-9983. Disponível em: http://cadernos.cenpec.org.br/cadernos/index.php/cadernos/article/view/293. Acesso em: 02 nov. 2020. DOI: http://dx.doi.org/10.18676/cadernoscenpec.v4i2.293.

SOUSA, G.C. de, GOMES, A.B. de A. Apoio à promoção de atividades históricas com tecnologia. Pesquisa, Sociedade e Desenvolvimento, v.9, n.5, p.1-14, 2020. DOI: https://doi.org/10.33448/rsd-v9i5.3206.

SOUSA, G.C. de, COSTA, A.E. J. da. Investigando a Conjunção entre História da Matemática e Tecnologia de Informação e Comunicação, Por Meio De Um Levantamento Bibliográfico Em Eventos Internacionais De Educação Matemática. Boletim Cearense de Educação e História da Matemática (BCEHM) v.4, n.11, p.621, 2017.

SOUSA, G.C. Uso da História da Matemática e Tecnologias de Informação e da Comunicação: Alianças Possíveis e Potenciais Para o Ensino de Matemática. Anais XII Encontro Nacional de Educação Matemática, p. 1-13, 2016. Disponível em: http://www.sbembrasil.org.br/enem2016/anais/pdf/5924_3414_ID.pdf. Acesso em: 02 nov 2020. 
Saberes docentes para a conjunção entre história da matemática e tecnologias digitais da informação e comunicação nas aulas de matemática: análise do potencial de um curso de extensão

SOUSA, Giselle Costa de. Aliança entre História da Matemática e Tecnologias via Investigação Matemática: reflexões e práticas. São Paulo: Editora Livraria da Física, 2020a.

SOUSA, Giselle Costa de. Aliança entre HM, TDIC e IM: fundamentos e aplicações. In: REMATEC: Revista de Matemática, Ensino e Cultura, Ano 15, Fluxo Contínuo, 2020b, p.117-136. Disponível em:

<http://www.rematec.net.br/index.php/rematec/article/view/239>. Acesso em: 27 ago. 2020.

TZANAKIS, C., \& THOMAIDIS, Y. Integrating the close historical development of mathematicsand physics in mathematics education: Some methodological and epistemological remarks. Forthe Learning of Mathematics, v.20, n.1, p.44-55, 2000.

VIEIRA, L. A. História da Matemática e Tecnologia da Informação e da Comunicação no Ensino de Função. Dissertação (Mestrado em Ensino de Ciências Naturais e Matemática) - Universidade Federal do Rio Grande do Norte. (UFRN), Natal. 2017, 249. f.

YOUSCHKEVITCH, A. P. The Concept of Function up to the Middle of the 19 th Century. Moscow: Institute for History of Science and Technology, 1976.

WICHNOSKI, P.; KLÜBER, T. E. Investigação Matemática: possíveis articulações com a História da Matemática, TIC e Resolução de Problemas. Educação Matemática Pesquisa, São Paulo, v.21, n.2, p. 539-561, 2019. Disponível em: revistas.pucsp.br/emp/article/download/42231/pdf. Acesso em: 02 nov. 2020.

Recebido em: 04 / 11 / 2020

Aprovado em: 19 / 11 / 2020 